\title{
A Robust Design Window for the Heliotron DEMO Reactors*)
}

\author{
Takuya GOTO, Junichi MIYAZAWA, Teruya TANAKA, Nagato YANAGI and Akio SAGARA \\ National Institute for Fusion Science, 322-6 Oroshi-cho, Toki, Gifu 509-5292, Japan
}

(Received 23 December 2010 / Accepted 21 March 2011)

\begin{abstract}
According to the achievements in the Large Helical Device (LHD) experiments, a design of a DEMO reactor with a LHD-type heliotron system is foreseeable. On the other hand, a DEMO is the next step reactor and high reliability and feasibility are demanded in its design. In this study, a robust design window, i.e., the design window that is not sensitive to a change in uncertain physics parameters, was surveyed through parametric scans using a system design code. It was found that a difference in main design parameters (major radius, magnetic field strength, fusion output) gives only a small change in a dependence of physics requirements on the physics conditions. Therefore, it is important to find the design window with a lower requirement on the confinement improvement to assure the design robustness. In this respect, a reduction in the minimum inboard blanket space, one of the key parameters in a LHD-type heliotron reactor design, can effectively expand the design window and contributes to the design robustness. An acceptance of higher neutron wall load and an achievement of further high confinement improvement are also expected to make both a DEMO and a commercial reactor to be more feasible and economically attractive.
\end{abstract}

(c) 2011 The Japan Society of Plasma Science and Nuclear Fusion Research

Keywords: heliotron system, DEMO reactor, design window analysis, system design code, blanket space

DOI: $10.1585 /$ pfr.6.2405083

\section{Introduction}

A helical system with net-current-free plasma has suitable properties for a DEMO and a commercial fusion reactor. Since it has no operational restriction due to a plasma current, a steady-state operation can be easily achieved. It also needs no current drive power and the self-ignition state is attainable, resulting in low recirculation power and high plant efficiency. Among the helical system, heliotron system with two continuous helical coils has recorded several remarkable achievements in the Large Helical Device (LHD) experiments [1]. LHD has achieved the reactor-level high-beta state ( $\sim 5 \%$ by volumeaveraged value) with the duration of much longer than energy confinement time $\left(\sim 100 \tau_{\mathrm{E}}\right)$. LHD has also achieved an extremely high central electron density with the internal diffusion barrier (IDB-SDC, $n_{\mathrm{e} 0}=1.2 \times 10^{21} \mathrm{~m}^{-3}$ ). A long time discharge for about 1 hour without any disruptive event has also been obtained. There are other several remarkable observations such as an impurity hole. A steady state divertor detach scenario by a noble gas puff and the utilization of an externally-induced magnetic island has also been proposed. In this respect, a LHD-type heliotron DEMO reactor is now foreseeable and its design feasibility study is quite meaningful.

Generally, the design point of DEMO and commercial reactors is determined through a kind of optimization process (e.g., minimization of the cost of electricity). There

author's e-mail: goto.takuya@LHD.nifs.ac.jp

*) This article is based on the presentation at the 20th International Toki Conference (ITC20). are, however, several uncertainties in the physics conditions of a burning plasma; density and temperature profiles, helium ash fraction, alpha heating efficiency, and so on. On the other hand, a DEMO is the next step reactor and high reliability and feasibility should be assured in its design. In this respect, it is important to find a robust design point, i.e., the design point which is not so much affected due to a change in the uncertain physics conditions, in place of finding the optimum design point but with many assumptions. Therefore, design window analyses including considerations of such uncertainties were carried out using a system design code for heliotron reactors.

\section{Design Window of a LHD-type He- liotron Reactor}

A design window of fusion reactors is determined by balancing various design factors. Here a brief review of the heliotron reactor design FFHR-2m2 [2] is given as an example. FFHR-2m 2 is a LHD-type heliotron reactor based on the LHD high-beta discharges. Therefore, it has the geometry which is basically a similar extension of that of LHD: two continuous helical coils with toroidal pitch number $m=10$, the inward-shifted magnetic configuration with a ratio between magnetic axis position and helical coil winding center position of $R_{\mathrm{ax}} / R_{\mathrm{c}}=3.6 / 3.9$, plasma aspect ratio of $A_{\mathrm{p}} \sim 6.6$. Since FFHR-2m 2 is designed as both a DEMO and a commercial reactor, a fusion power output of $P_{\text {fus }}=3 \mathrm{GW}$ is assumed to realize the net electric output comparable to the current large-scale power plants $\left(P_{\mathrm{e}, \text { net }} \sim 1 \mathrm{GWe}\right)$. The average neutron wall load is set to be 


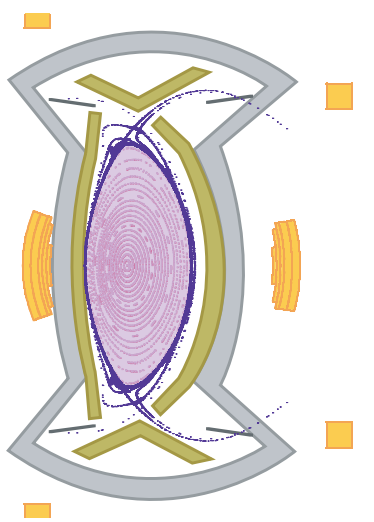

Fig. 1 Cross-sectional view of a LHD-type heliotron system on the vertically elongated poloidal cross-section.

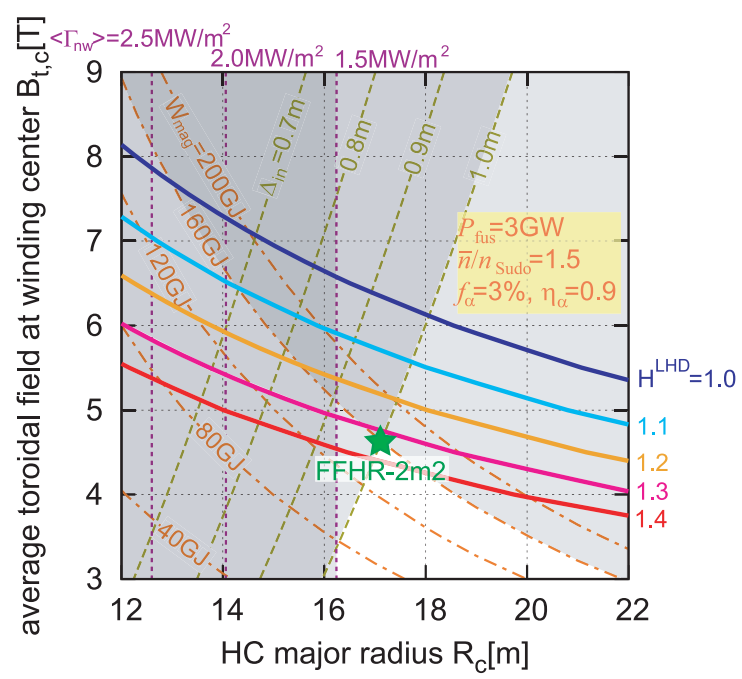

Fig. 2 Contour map of the engineering constraints and the required confinement improvement for a LHD-type heliotron reactor with $3 \mathrm{GW}$ fusion output. The shaded region corresponds to the design region that does not satisfy the engineering constraints. The candidate design point of FFHR-2m2 is plotted as the star symbol.

$\leq 1.5 \mathrm{MW} / \mathrm{m}^{2}$ to suppress neutron damage on the structural material of the blanket system under 100 dpa after a 30 full power years (FPYs) operation with the long-life blanket concept [3]. A stored magnetic energy $W_{\text {mag }}$ is an index of the total mass amount of the superconducting magnet system including the supporting structure. The superconducting magnet system with $W_{\text {mag }}=120-140$ GJ can be constructed with a small extension of the ITER-relevant technology [4] and the achievable maximum value is estimated to be $\sim 160 \mathrm{GJ}$. Therefore, $W_{\text {mag }} \leq 160 \mathrm{GJ}$ is assumed in the design of FFHR-2m2. In a LHD-type heliotron system, the space between the helical coil and the plasma confinement region including ergodized layers has its minimum at the inboard side on the vertically elongated poloidal crosssection as shown in Fig. 1. On the other hand, blanket system with a thickness of $\sim 1 \mathrm{~m}$ is required to assure the sufficient tritium breeding ratio (TBR) of $>1.05$ and the ra- (a)

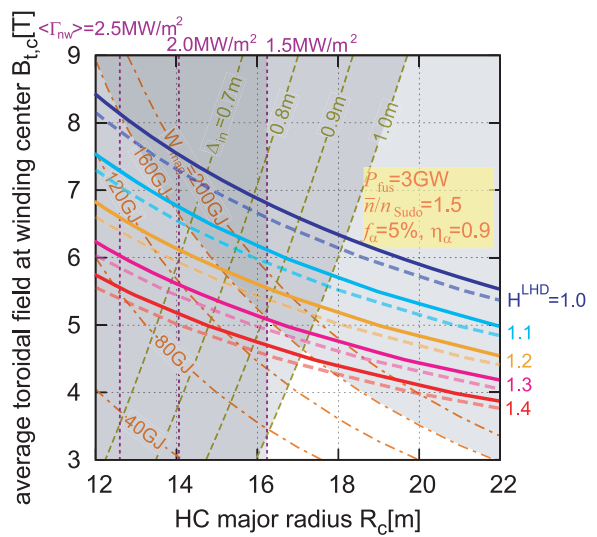

(b)

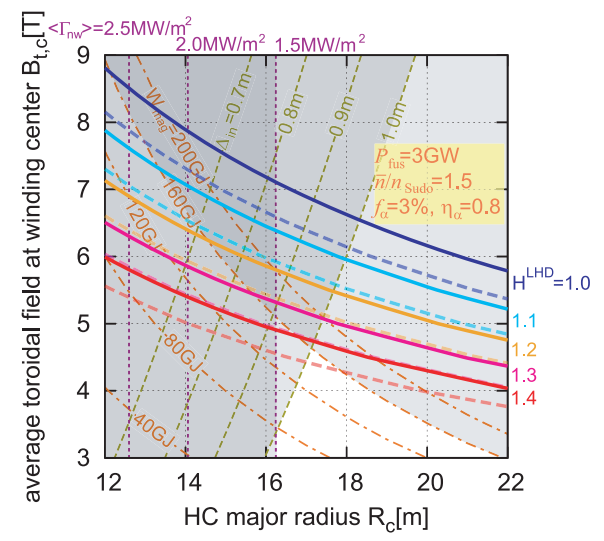

(c)

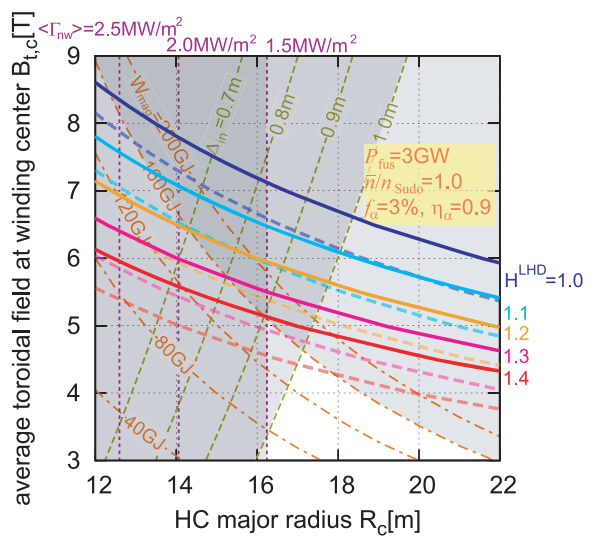

Fig. 3 Dependence of the required confinement improvement on (a) helium ash fraction (from 3\% to 5\%), (b) alpha heating fraction (from $90 \%$ to $80 \%$ ), (c) line-averaged electron density (from 1.5 to 1.0 times Sudo density scaling). Dotted lines in each figure correspond to the reference case (the same in Fig. 2).

diation shielding for the superconducting coils [2]. In the design of FFHR-2m2, the blanket thickness at the all positions including the minimum position is set to be $\geq 1.0 \mathrm{~m}$.

Figure 2 shows how these engineering constraints determine the design window on $R_{\mathrm{c}}-B_{\mathrm{t}, \mathrm{c}}$ plane ( $B_{\mathrm{t}, \mathrm{c}}$ is the helical coil major radius and the toroidal field at the helical coil winding center averaged over one helical pitch). In the case of a similar extension of the LHD, the inboard 
minimum blanket space $\Delta_{\text {in }}$ is proportional to $R_{\mathrm{c}}$. In the case that the current density of helical coils $j_{\mathrm{c}}$ is kept $\left(j_{\mathrm{c}}=\right.$ $25 \mathrm{~A} / \mathrm{mm}^{2}$ has been assumed), the contours of $\Delta_{\text {in }}$ becomes upward-sloping curves. The stored magnetic energy $W_{\text {mag }}$ is proportional to $R_{\mathrm{c}}^{3} B_{\mathrm{t}, \mathrm{c}}^{2}$. Note that these constraints are fixed regardless of plasma conditions. The constraint due to the average neutron wall load $\left\langle\Gamma_{\mathrm{nw}}>\right.$ depends on the fusion output. In the case of $P_{\text {fus }}=3 \mathrm{GW}$, the possible design window for FFHR-2m2 is described as the area which is not shaded in Fig. 2. Therefore, the design point of $R_{\mathrm{c}}$ $=17 \mathrm{~m}$ and $B_{\mathrm{t}, \mathrm{c}}=4.7 \mathrm{~T}$ has been selected as a candidate for FFHR-2m2. In the FFHR-2m 2 design, the following physics conditions has been assumed; line-averaged electron density of 1.5 times Sudo density limit [5], helium ash fraction of $f_{\alpha}=3 \%$, and alpha heating efficiency of $\eta_{\alpha}=90 \%$. Under these assumptions, the required confinement improvement from the present experiments $(0.93$ times ISS04v3 scaling [6]) $H^{\text {LHD }}=1.3$ enables the design point of FFHR-2m2 with satisfying the self-ignition condition.

It was found that, however, $H^{\mathrm{LHD}}$ is significantly affected by the assumed physics conditions. Figure 3 shows a dependence of $H^{\text {LHD }}$ on the three physics conditions: $f_{\alpha}$, $\eta_{\alpha}$ and the ratio between the line-averaged electron density and Sudo density limit. In the all cases $H^{\mathrm{LHD}}$ increases by $10-20 \%$ irrespective of the values of both $R_{\mathrm{c}}$ and $B_{\mathrm{t}, \mathrm{c}}$. It was also found that a difference in $P_{\text {fus }}$ gives only a small change in the sensitiveness of $H^{\mathrm{LHD}}$ to the physics conditions. Consequently, it is important to find the design window that allows a lower $H^{\mathrm{LHD}}$, a larger margin for unexpected changes in the physics conditions, to assure the design robustness.

\section{Flexible Design Concept for DEMO}

To find a robust and a more attractive design window, the design window analyses including a reconsideration of the engineering constraints were carried out. The first priority of a DEMO reactor is a demonstration of a steadystate, self-ignition plasma operation accompanying a plantscale net electric power generation. It should be noted that a DEMO is the first reactor that generates fusion neutrons, energetic particles, and high heat flux at the same time. Therefore the integrity of engineering components (e.g., divertor, blanket) should be confirmed in the operation of a DEMO reactor itself. To achieve these purposes, a longterm ( 30 FPYs) operation and a fusion output of $3 \mathrm{GW}$ are not a necessary condition of a DEMO reactor. The minimum inboard blanket thickness also can be reduced. Figure 4 shows the distribution of fast $(>0.1 \mathrm{MeV})$ neutron flux in the blanket system proposed in the design study of FFHR-2m 2 with $P_{\text {fus }}=3 \mathrm{GW}$. The material used for superconducting coils could endure the fast neutron fluence of $\sim 10^{19} \mathrm{n} / \mathrm{cm}^{2}$. Therefore, in the case of 30 FPYs operation, the fast neutron flux at the front of the helical coil must be suppressed $<10^{10} \mathrm{n} / \mathrm{cm}^{2} / \mathrm{s}$. In the design study of

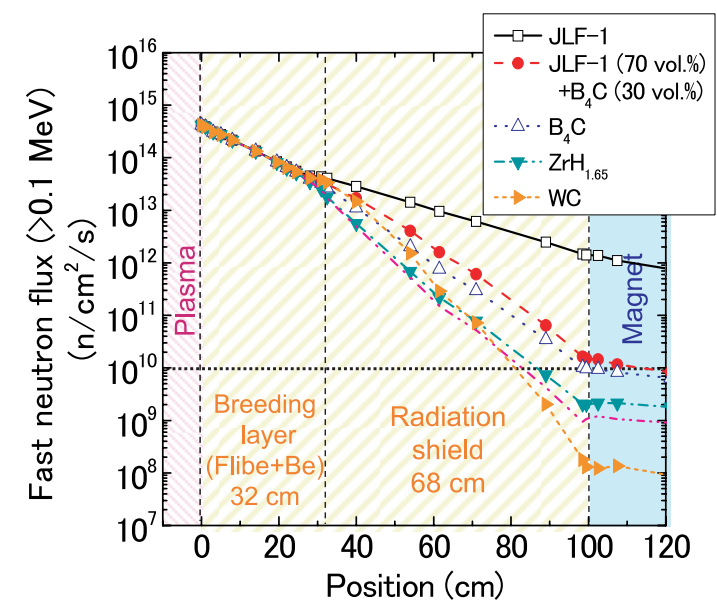

Fig. 4 Distributions of fast $(>0.1 \mathrm{MeV})$ neutron flux in the FFHR blanket system with different materials composition in radiation shield. The dotted horizontal line $\left(10^{10} \mathrm{n} / \mathrm{cm}^{2} / \mathrm{s}\right)$ is the flux limit on the superconducting magnet in the case of a 30 FPYs operation.

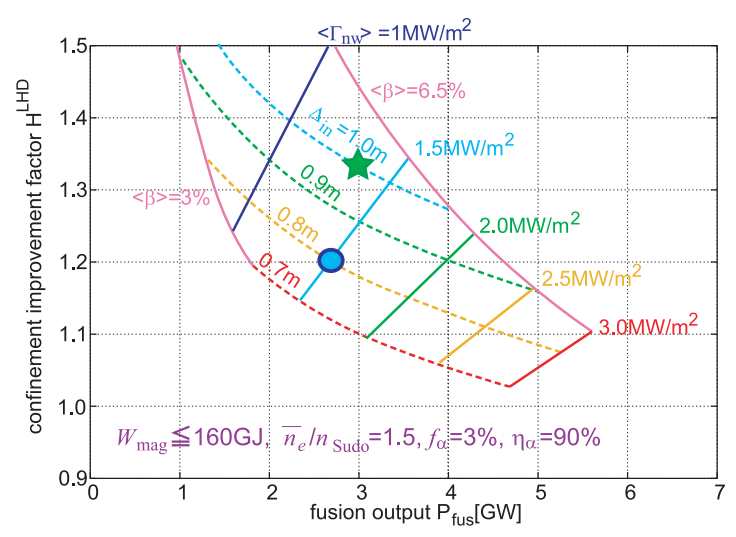

Fig. 5 Dependence of the required confinement improvement on the fusion power with various constraints on the minimum inboard blanket space $\Delta_{\text {in }}$ and the averaged neutron wall load $\left\langle\Gamma_{\mathrm{nw}}\right\rangle$. The star symbol denotes the design point of FFHR-2m2. The circle is the possible design point with $\Delta_{\text {in }}=0.8 \mathrm{~m}$ and the same $<\Gamma_{\mathrm{nw}}>$ as that of FFHR-2m2 $\left(1.5 \mathrm{MW} / \mathrm{m}^{2}\right)$.

FFHR-2m2, the reduction of the blanket thickness by thinning the radiation shield layer using tungsten carbide has already been proposed [2]. But the reflection of the fast neutrons from the magnet was not considered. Figure 4 is the re-evaluation result and shows that advanced materials such as zirconium hydride or tungsten carbide can effectively slow down the fast neutrons and the thickness of the radiation shield can be reduced by $\sim 20 \mathrm{~cm}$. If the operational period is shortened, higher fast neutron flux on the superconducting coil is acceptable and the radiation shield thickness can also be reduced.

The reduction in the inboard minimum blanket thickness effectively expands the design window. Figure 5 shows the design window with the same physics condi- 


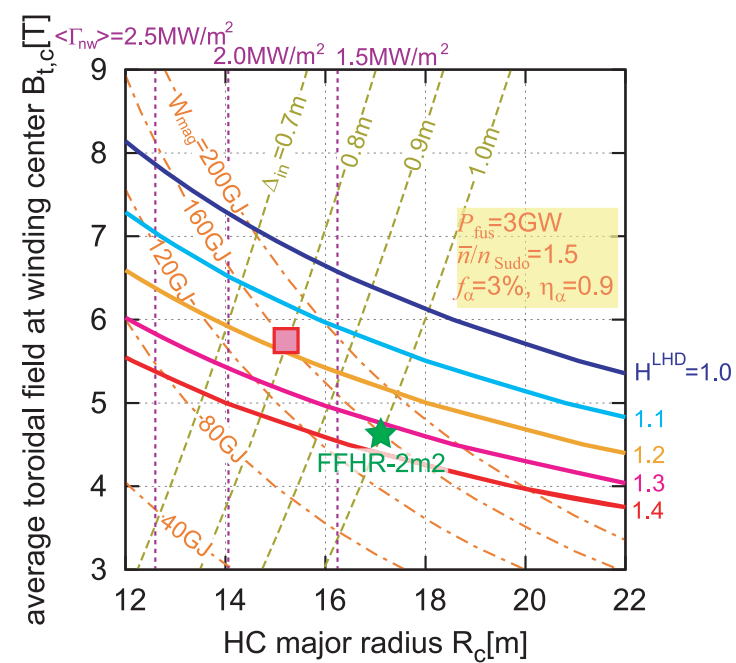

Fig. 6 Design window of a LHD-type heliotron DEMO reactor with the same assumptions in the physics conditions and fusion power as those of FFHR-2m2. The square corresponds to the design point with $\Delta_{\text {in }}=0.8 \mathrm{~m}$, which can be selected if $\left\langle\Gamma_{\mathrm{nw}}\right\rangle=1.75 \mathrm{MW} / \mathrm{m}^{2}$ is accepted.

tions and the constraint on the stored magnetic energy as assumed in the design of FFHR-2m 2 . In the case of $\Delta_{\text {in }}=$ $0.8 \mathrm{~m}$, the design points with $H^{\mathrm{LHD}} \sim 1.2$ can be selected with keeping $\left\langle\Gamma_{\mathrm{nw}}\right\rangle \leq 1.5 \mathrm{MW} / \mathrm{m}^{2}$ by reducing a fusion power to $2.5 \mathrm{GW}$. If higher $\left\langle\Gamma_{\mathrm{nw}}\right\rangle$ is accepted, $H^{\mathrm{LHD}}$ can be reduced further by increasing a fusion power. Figure 6 shows the design window on $R_{\mathrm{c}}-B_{\mathrm{t}, \mathrm{c}}$ plane for the latter case. In this case, the design point with $R_{\mathrm{c}} \sim 15 \mathrm{~m}$ and $B_{\mathrm{t}, \mathrm{c}} \sim 6 \mathrm{~T}$ can be selected, which realizes $H^{\mathrm{LHD}}<1.2$ with keeping $P_{\text {fus }}=3 \mathrm{GW}$ and $W_{\text {mag }}=160 \mathrm{GJ}$. Conversely, $W_{\text {mag }}$ can be reduced by reducing magnetic field strength if the higher $H^{\mathrm{LHD}}$ is achieved, resulting in an enhancement of the design feasibility of the superconducting magnet system and a reduction in the construction cost.

The reduction in the minimum inboard blanket space has other effects. It allows a reduction in the current density of helical coils. It also enables a flexible selection of the magnetic configuration (e.g., smaller plasma aspect ra- tio). Such increase in the design margin also contributes to the design robustness of a DEMO reactor.

\section{Conclusion}

In order to find a robust design window of a LHDtype heliotron DEMO reactor, parametric scans were carried out using a system design code for heliotron reactors. It was found that a difference in the main design parameters (major radius, magnetic field strength, fusion output) gives only a small change in a dependence of the physics requirements (especially the required confinement improvement) on the physics conditions. Therefore it is important to find a design window with a lower requirement on the confinement improvement, which has a larger margin for unexpected changes in the physics conditions, in order to assure the design robustness of a heliotron DEMO reactor. In this respect, a reduction in the minimum inboard blanket thickness is effective and technologically achievable. The simultaneous achievements of a reduction in the blanket thickness and an acceptance of higher averaged neutron wall loading enables a relaxation in the physics requirements with keeping the other design parameters. This effect can also expand the design window of a commercial reactor. Consequently, the progress in the engineering research which enables a reduction of the blanket space (e.g., an adoption of advanced materials, an optimization of the inner-structure of the blanket system, etc.) is strongly expected. If the magnetic field strength can be reduced, the design feasibility of the superconducting magnet system is enhanced and the total plant capital cost can be reduced. Therefore, further improvement in the plasma confinement property is also expected to make a DEMO and a commercial reactor to be more economically attractive one.

[1] A. Komori et al., Fusion Sci. Technol. 58, 1 (2010).

[2] A. Sagara et al., Fusion Eng. Des. 83, 1690 (2008).

[3] A. Sagara et al., Nucl. Fusion 45, 258 (2005).

[4] S. Imagawa et al., Nucl. Fusion 49, 075017 (2009).

[5] S. Sudo et al., Nucl. Fusion 30, 11 (1990).

[6] H. Yamada et al., Nucl. Fusion 45, 1684 (2005). 